\title{
The National Nanotechnology Infrastructure Network's Education and Outreach Programs - Understanding Size and Scale
}

\author{
Nancy Healy
}

Georgia Institute of Technology and National Nanotechnology Infrastructure Network 791 Atlantic Dr. NW, Atlanta, GA 30332

Nanotechnology is considered by many to be the next "industrial revolution." The NSF estimates that by 2015 nanoscale science and engineering will be $\$ 3$ trillion industry with the U.S. needing approximately 1 million workers. Workforce development programs are needed to excite students about possible education and career opportunities to ensure that the U.S. maintains its competitive edge in this fast-growing field. The National Nanotechnology Infrastructure Network (NNIN) is an integrated geographically-diverse partnership of 14 university-based laboratories supported by the National Science Foundation. The primary focus of NNIN is to serve as state-of-the-art resource centers for researchers. The NNIN also has extensive education outreach programs for the K-gray population.

The purpose of this presentation will be twofold: 1. provide an overview of the NNIN's education and outreach programs and 2. provide information on how we are teaching students about size and scale. Nanotechnology is science and engineering that occurs at the dimensions of 1-100 nanometers (in one dimension). At this scale, unique phenomena occur in materials which lead to researchers developing novel applications. At the nanoscale, materials may behave differently than the bulk counterpart. Nanotechnology involves "imaging, measuring, modeling, and manipulating matter at this length scale."[1]

A nanometer is one-billionth of a meter or $1 \times 10^{-9} \mathrm{~m}$. The average human hair is approximately 50,000 to $70,000 \mathrm{~nm}$ in diameter. The concepts of size and scale are very important to understand when discussing nanotechnology because size defines the nanoscale and at the nanoscale the behavior of matter varies.

We have found that most students can provide the various SI units of measurement and may even define these prefixes. But where most students have difficulty, is understanding differences in size and scale as materials move from macro to micro to nano scales. We utilize a variety of activities to help students develop an understanding of size and scale and how small objects in the nanoworld actually are. We employ two tools of nano, SEM and AFM, to allow students to actually explore the nanoworld. One approach that we have taken is to have students use a variety of optical devices magnifiers, DinoLite ${ }^{\mathrm{TM}}$, optical microscope - to view everyday materials. Then, we have these materials imaged by the SEM and AFM. The AFM presents a challenge in that many students believe that they are "seeing" the nanoscale rather than seeing a graphic representation of the forces at the nanoscale. A correct understanding of this concept is very important for any one who uses the AFM as a teaching tool.

Our programs are designed to help students understand concepts relating to size and scale because of their importance in eventually allowing students to understand nanoscale phenomena. Size and scale 
play an important role in scientific research in that one must be able to decide on the correct tool or methodology to use at varying scales.

References

[1] National Nanotechnology Initiative, What is Nanotechnology? accessed at: http://www.nano.gov/html/facts/whatIsNano.html

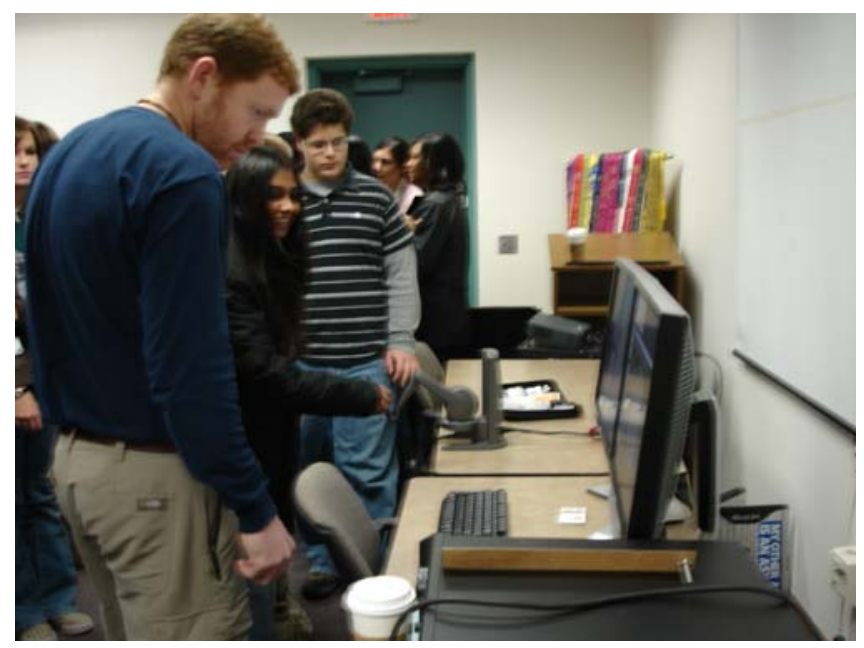

FIG. 1. Students from Gwinnett School for Science, Math, and Technology explore the NanoManipulator with staff from Asylum Research at a Georgia Tech event.

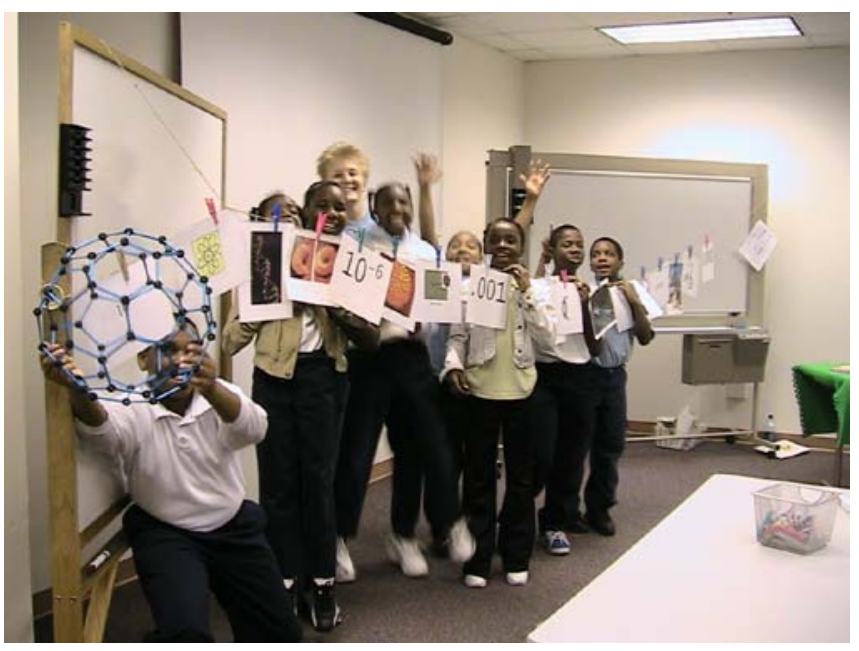

FIG. 2. Students from Centennial Park Elementary finish a size and scale activity at Georgia Tech. 